\title{
TurbuStat: Turbulence Statistics in Python
}

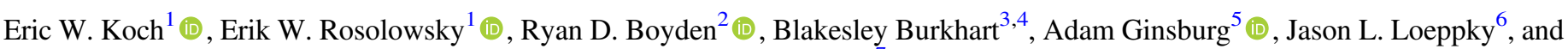 \\ Stella S. R. Offner ${ }^{7}$ (iD \\ ${ }^{1}$ University of Alberta, Department of Physics 4-183 CCIS, Edmonton AB T6G 2E1, Canada; koch.eric.w@gmail.com \\ ${ }^{2}$ Department of Astronomy and Steward Observatory, University of Arizona, 933 North Cherry Avenue, Tucson, AZ 85721, USA \\ ${ }^{3}$ Center for Computational Astrophysics, Flatiron Institute, 162 Fifth Avenue, New York, NY 10010, USA \\ ${ }^{4}$ Department of Physics and Astronomy, Rutgers, The State University of New Jersey, 136 Frelinghuysen Road, Piscataway, NJ 08854, USA \\ ${ }^{5}$ Jansky fellow of the National Radio Astronomy Observatory, 1003 Lopezville Road, Socorro, NM 87801, USA \\ ${ }^{6}$ Department of Physics, University of British Columbia, Okanagan Campus, 3333 University Way, Kelowna, BC V1V 1V7, Canada \\ ${ }^{7}$ Department of Astronomy, The University of Texas at Austin, 2515 Speedway, Stop C1400, Austin, TX 78712, USA \\ Received 2019 March 4; revised 2019 April 21; accepted 2019 April 22; published 2019 June 5
}

\begin{abstract}
We present TURBUSTAT (v1.0): a PYTHON package for computing turbulence statistics in spectral-line data cubes. TURBUSTAT includes implementations of 14 methods for recovering turbulent properties from observational data. Additional features of the software include: distance metrics for comparing two data sets; a segmented linear model for fitting lines with a break point; a two-dimensional elliptical power-law model; multicore fast-Fourier-transform support; a suite for producing simulated observations of fractional Brownian Motion fields, including twodimensional images and optically thin $\mathrm{H}$ I data cubes; and functions for creating realistic world coordinate system information for synthetic observations. This paper summarizes the TURBUSTAT package and provides representative examples using several different methods. TURBUSTAT is an open-source package and we welcome community feedback and contributions.
\end{abstract}

Key words: methods: data analysis - methods: statistical - turbulence

Supporting material: data behind figure

\section{Introduction}

Turbulence is ubiquitous throughout the interstellar medium (ISM; see reviews by Elmegreen \& Scalo 2004; Lazarian 2009). Observations of different ISM phases demonstrate that similar turbulent properties are found over a wide range of scales (e.g., Armstrong et al. 1995; Chepurnov \& Lazarian 2010). Connecting these observations with theoretical and numerical predictions is critical for understanding how turbulence affects the structure and motion of the ISM. This connection provides important inputs for many astrophysical processes; for example, turbulence plays a central role in modern star formation theories (e.g., Krumholz et al. 2009; Ostriker et al. 2010; Federrath \& Klessen 2012; Burkhart 2018).

Determining turbulent properties from observations is challenging because of the limited information available and the complexity of the ISM. Two-dimensional images, in particular column density or extinction maps, are a projection of a threedimensional turbulent field. Spectral line observations provide additional constraints from the line-of-sight velocity, though features resolved in velocity may not be spatially distinct (Beaumont et al. 2013; Burkhart et al. 2013b) and are affected by opacity and line excitation (Burkhart et al. 2013a; Correia et al. 2016). This means that observations miss up to four of the full six-dimensional phase space ${ }^{8}$ that describe the ISM structure and its motion. The inherent complexity of the ISM adds to the difficulty in interpreting observations. Turbulence in the ISM may be driven by multiple energy injection sources on different scales (McKee \& Ostriker 2007; Krumholz et al. 2014, 2018; Chepurnov et al. 2015) and is affected by variations in magnetic field strength and orientation (Goldreich \& Sridhar 1995; Cho \& Lazarian 2003; Burkhart et al. 2009, 2015b;

\footnotetext{
Three spatial and three velocity dimensions.
}

Meyer et al. 2014; Hull et al. 2017). There are additional physical effects at work, including phase transitions that affect thermodynamic properties and gravitational collapse within molecular clouds (Bialy et al. 2017; Hill et al. 2018).

Significant effort over the last $\sim 40 \mathrm{yr}$ has sought to connect predicted turbulent properties from theory (e.g., Goldreich \& Sridhar 1995) with (magneto-)hydrodynamic simulations (e.g., Cho \& Lazarian 2003; Kowal et al. 2007; Kritsuk et al. 2007; Federrath et al. 2008; Collins et al. 2012) and observations (e.g., Burkhart et al. 2010). To make these connections, an array of methods have been proposed in the literature to recover turbulent properties from observational data. These methods utilize either two-dimensional images or three-dimensional spectral-line data cubes. ${ }^{9}$ The latter has been of particular interest for recovering properties of the turbulent density and velocity fields (e.g., Lazarian \& Pogosyan 2000).

We have developed TURBUSTAT, a publicly available Python package that implements 14 observational diagnostics of ISM turbulence described in the literature. TURBUSTAT provides a common framework for running and comparing turbulence diagnostics, including comparisons between simulations and observations (e.g., Boyden et al. 2016, 2018; Koch et al. 2017; Haworth et al. 2018). The use of some techniques has been limited by the lack of a publicly available implementation. Furthermore, many studies focus on using one or a small number of techniques. This has resulted in a limited understanding of the regimes where particular methods are best suited and the limits where inherent assumptions in a method break down. The breadth of techniques in TURBUSTAT provides the opportunity to explore these issues.

\footnotetext{
9 Observations of polarization provide additional information (e.g., Gaensler et al. 2011; Iacobelli et al. 2014; Herron et al. 2018), but TURBUSTAT does not currently handle polarization data.
} 
In this paper, we present an overview of TURBUSTAT's first major release (v1.0), including a description of the methods implemented in TURBUSTAT (Section 2), an overview of the package (Section 3), and a demonstration of TURBUSTAT's capabilities with representative examples (Section 4). The Appendices highlight the choice of normalization for the wavelet transform (Gill \& Henriksen 1990, Appendix A), a comparison of our delta-variance implementation to the original IDL code (Ossenkopf et al. 2008a; Appendix B), and a series of memory and timing tests for the methods in TURBUSTAT (Appendix C). TURBUSTAT is an open source and includes extensive documentation and tutorials on the use of the methods. ${ }^{10} \mathrm{We}$ encourage feedback from the community and welcome contributions.

\section{Methods}

TURBUSTAT (v1.0 Koch et al. 2019) has implementations of 14 literature methods ${ }^{11}$ that recover properties related to turbulence from observational data. We briefly describe the methods and relevant literature here (also see descriptions in Boyden et al. 2016, 2018; Koch et al. 2017), and note that the package documentation contains thorough explanations and code examples.

\subsection{Structure Analysis}

The spatial structure of the ISM is hierarchical. Statistics that characterize the structural properties of an image or spectralline data cube are one way to describe the hierarchical structure. TURBUSTAT has two methods that provide a nonparametric description of hierarchical structure.

Genus. Genus statistics are a measure of topology. The value of the genus statistic is the difference between the number of isolated regions above and below a threshold. A genus curve is produced by varying the threshold over a range of values. The first use of the genus statistic on column density maps was introduced Lazarian et al. (2002) and later expanded on by Kowal et al. (2007), Chepurnov et al. (2008), and Burkhart et al. (2012). The implementation in TURBUSTAT closely follows the approach from Chepurnov et al. (2008).

Dendrograms. Dendrograms are a common method for exploring the hierarchical structure of data. Their use in molecular cloud studies was proposed by Rosolowsky et al. (2008) and Goodman et al. (2009), where pixels in an image or data cube are combined into hierarchical clusters based on their brightness (also see Houlahan \& Scalo 1990, 1992). Burkhart et al. (2013) explored two statistics based on the dendrogram structure: (1) the relation between the number of structures in the dendrogram as a function of the branch height, and (2) the histogram of peak intensity in each structure of the dendrogram. Dendrograms can also be combined with other statistics, such as 1D probability distribution functions (PDFs; Chen et al. 2018). TURBUSTAT implements both of these statistics and utilizes astrodendro ${ }^{12}$ to compute the dendrograms.

\subsection{Properties of Turbulence}

Despite the complexity of astrophysical turbulence, several statistics for observational data have theoretically motivated

\footnotetext{
10 turbustat.readthedocs.io

11 We note that this number differs from Boyden et al. $(2016,2018)$ since (i) the Tsallis statistic was not used and (ii) multiple outputs of dendrograms and statistical moments were counted as individual methods.

12 dendrograms.readthedocs.io
}

properties. TURBUSTAT implements a number of these methods, particularly those related to power spectra.

Spatial Power Spectrum (SPS). The SPS is a widely used method for finding the turbulent field index in the ISM. The power-spectrum index from the column density or velocity centroid map is related to the underlying density or velocity field of the ISM, respectively (e.g., Lazarian \& Pogosyan 2000; Esquivel \& Lazarian 2005). Most studies reduce the 2D power spectrum of the image into one-dimension with azimuthalaveraging then fit a power law to find the index (e.g., Crovisier \& Dickey 1983; Scalo 1984; Stanimirović \& Lazarian 2001; Padoan et al. 2006; Burkhart et al. 2013a; Pingel et al. 2018). Additional information can be retained when modeling the full 2D power spectrum, including preferred directions of structure in the image and anisotropy (e.g., Martin et al. 2015; Kalberla \& Kerp 2016) that are predicted to relate to the magnetic field structure (Burkhart et al. 2014; González-Casanova \& Lazarian 2017; Kandel et al. 2017). TURBUSTAT can be used for both types of studies and can model breaks in the power spectrum and the effect of a telescope beam (see Section 3.6).

Modified Velocity Centroids (MVCs). MVC is an adaptation of the SPS introduced by Lazarian \& Esquivel (2003) that accounts for velocity-density correlations, which alter the power spectrum of a velocity centroid map. The centroid power spectrum is corrected by subtracting the column density power spectrum multiplied by the average velocity dispersion in the data. Esquivel \& Lazarian (2005) explore the limits for when this correction is required. The MVC implementation in TURBUSTAT requires an input of the velocity centroid, integrated intensity, ${ }^{13}$ and line width ${ }^{14}$ maps. The formulation in Lazarian \& Esquivel (2003) requires unnormalized velocity centroids (i.e., without dividing by the integrated intensity). Since most observational products do not utilize this form of centroid, our implementation converts the centroid map to an unnormalized form. TURBUSTAT's implementation of MVC has the same features described for the SPS.

Velocity Channel Analysis (VCA). Lazarian \& Pogosyan (2000) show that, by considering the power spectrum of a spectral-line cube integrated over spectral frequencies, the index of the SPS will be altered by velocity fluctuations for sufficiently small spectral channel widths (see also Lazarian \& Pogosyan 2004). By increasing the spectral channel width, the power-spectrum index approaches the power spectrum of the column density, which is set only by density fluctuations (e.g., Stanimirović \& Lazarian 2001; Muller et al. 2004). Recent work by Kandel et al. (2016) expands these theoretical predictions to include anisotropy in $2 \mathrm{D}$ power spectra. The VCA implementation in TURBUSTAT has the same functionality as the SPS and includes the ability to alter the spectral channel width of a given cube (see Section 4.1.2).

Velocity Coordinate Spectrum (VCS). The VCS is a complementary technique to VCA that is first mentioned in Lazarian \& Pogosyan (2000) and later expanded on in Lazarian \& Pogosyan (2006; also see Lazarian \& Pogosyan 2008; Chepurnov \& Lazarian 2009). While VCA integrates over the spectral dimension, VCS is the integration over the spatial dimensions, which yields a 1D spectral power spectrum. The VCS implementation in TURBUSTAT fits a broken linear model to the VCS (Section 3.6), mimicking the asymptotic high- and low-resolution solutions presented in Lazarian \& Pogosyan (2006).

\footnotetext{
13 Or column density.

14 From the second moment.
} 
A future extension of the code will include fitting with the complete VCS model (Chepurnov et al. 2010, 2015), which can, for example, constrain the turbulent driving scale.

Bispectrum. The bispectrum is the Fourier transform of the three-point correlation function, and is the next-order analog to the power spectrum. Unlike the power spectrum, the bispectrum includes phase information, allowing for correlations between different spatial frequencies to be explored. Burkhart et al. (2009) explored how these correlations change in different MHD regimes and later extended the study to consider the HI column density of the Small Magellanic Cloud (Burkhart et al. 2010). Quantitative comparisons between bispectra are more difficult than with the power spectrum, which can be characterized only by its index. The bispectrum is a complex quantity and cannot generally be reduced to a $1 \mathrm{D}$ representation. Instead, the bicoherence- $-\mathrm{a}$ real-valued normalized quantity that represents phase coupling - can be calculated. The TURBUSTAT implementation includes both quantities, using the bicoherence definition from Hagihira et al. (2001). Calculating the bispectrum for even a small image is expensive and time consuming. Our implementation uses Monte Carlo sampling, with user input on the number of samples, to compute the bispectrum for each combination of wavenumbers. A future extension to avoid this sampling is to utilize the multi-pole expansion introduced recently by Portillo et al. (2018).

Wavelet Transform. Gill \& Henriksen (1990) measure the amount of structure as a function of spatial scale by taking the sum of positive values in a wavelet decomposition of a 2D image. This gives a transform that is similar to the structure function (e.g., Miesch \& Bally 1994). The wavelet implementation in TURBUSTAT is similar to the algorithm from Gill \& Henriksen (1990), though we introduce a change in the normalization of the wavelet kernels (Appendix A).

Delta Variance. Similar to the Gill \& Henriksen (1990) wavelet transform, ${ }^{15}$ the delta-variance technique is based on a wavelet decomposition, and is an extension of Allan Variance for a 1D time series (Stutzki et al. 1998; Bensch et al. 2001; Ossenkopf et al. 2001). The delta variance characterizes the image structure at a set of spatial scales by calculating the variance in the wavelet decomposition. An extension of this method for irregularly shaped observational maps was developed by Ossenkopf et al. (2008a, 2008b), which we have implemented in TURBUSTAT (see Appendix B for a comparison with the IDL code provided by these authors).

Principal Component Analysis (PCA). The PCA is a general dimensionality reduction procedure that identifies correlated components based on an orthogonal decomposition of a covariance matrix. Heyer \& Schloerb (1997) first applied a PCA to a spectral-line data cube by creating a covariance matrix of spectral channels in a data cube, decomposing that covariance matrix, and using the eigenvectors and eigenimages to recover characteristic spectral and spatial scales in the data. Combining these scales over the first $N$ eigenvalues produces a size-line width relation for the data, whose index can be related to the theoretically expected turbulent regimes. This technique was further developed in Brunt \& Heyer (2002a, 2002b) and RomanDuval et al. (2011), with an extension for measuring anisotropy (Heyer et al. 2008). An analytic model is presented in Brunt \& Heyer (2013). TURBUSTAT implements the algorithm described

\footnotetext{
15 Also see Zielinsky \& Stutzki (1999).
}

in Brunt \& Heyer (2002a, 2002b), including correction factors for the beam size and empirically derived calibrations (see Section 4.1.3). The spectral and spatial scales can be fit with orthogonal distance regression or with a Bayesian approach, both of which handle errors in both dimensions.

Spectral Correlation Function (SCF). The SCF was introduced by Rosolowsky et al. (1999) to relate spatial and spectral similarities of a data cube (see also Padoan et al. 2001, 2003). TURBUSTAT implements the form from Yeremi et al. (2014), where the statistic is the normalized rms difference between the cube and its spatially shifted self. By iterating over a range of spatial shifts in both spectral dimensions, we create a 2D correlation surface whose azimuthally averaged index has been shown to be sensitive to changes in turbulent properties (Padoan et al. 2003; Muller et al. 2004; Gaches et al. 2015). Our implementation can model the correlation surface in either one- or two-dimensions using a similar approach described for the SPS.

\subsection{Analysis of Distributions}

The distribution of values within a data set, or portions of a data set, are useful diagnostics in many settings. TURBUSTAT provides a convenient implementation for fitting probability distribution functions, and descriptions of distribution shapes.

Probability Distribution Functions (PDF). The most commonly used analysis technique to describe turbulent properties from observational data products is the PDF. Extensive work on PDFs from simulations (Vazquez-Semadeni 1994; Ostriker et al. 2001; Kowal et al. 2007; Federrath et al. 2008; Burkhart et al. 2009, 2017, e.g.,) and observations (e.g., Miesch \& Scalo 1995; Burkhart et al. 2010; Lombardi et al. 2015; Imara \& Burkhart 2016; Bialy et al. 2017) has provided a solid framework connecting the PDF to turbulent properties. The TURBUSTAT PDF implementation was written to emphasize flexibility in treatment and modeling of PDFs. Both images and cubes can be used, and the code can be used to quickly recover properties of the PDF and its empirical cumulative distribution function. The implementation utilizes the SCIPY.STATS ${ }^{16}$ continuous distributions for modeling, including normal, lognormal, and power-law distributions. A maximum-likelihood estimator is used to fit model distributions to the data.

Statistical Moments. An extension to PDF studies is an analysis of higher-order moments, namely the skewness and kurtosis, which are non-Gaussian indicators. Previous studies have explored skewness and kurtosis for column density, velocity centroid, and line width PDFs of simulations and observations (e.g., Padoan et al. 1999; Kowal et al. 2007; Burkhart et al. 2009, 2013a, 2015a). Burkhart et al. (2010) extend this approach using a rolling circular filter to create spatial moment maps of the $\mathrm{HI}$ in the SMC. This allows for spatial variations in the PDF moments to be explored. The TURBUSTAT implementation provides both methods.

Tsallis Statistics. The Tsallis distribution was introduced by Tsallis (1988) for describing multi-fractal systems. Esquivel \& Lazarian (2010) first introduced Tsallis statistics for modeling ISM turbulence (see also Tofflemire et al. 2011; Burkhart et al. 2013b, 2015a; González-Casanova et al. 2018). Our implementation of Tsallis statistics follows Esquivel \& Lazarian (2010) and uses a q-Gaussian distribution to model the difference in a given image as a function of spatial scale.

\footnotetext{
16 docs.scipy.org/doc/scipy/reference/stats.html
} 


\section{Overview of TURBUStat}

TURBUSTAT contains submodules for the computation of the methods, calculating moment arrays and their uncertainties from data cubes, and basic I/O operations for handling FITS files. In this section, we present the methods and other helpful utilities implemented in TURBUSTAT.

\subsection{Package Dependencies}

TURBUSTAT utilizes several packages in the python scientific computing infrastructure, namely built on numpy ${ }^{17}$ (Oliphant 2006), scipy ${ }^{18}$ (Jones et al. 2001), and matplotlib ${ }^{19}$ (Hunter 2007). We use astropy ${ }^{20}$ (Astropy Collaboration et al. 2018) for I/O operations, unit handling, convolution, and World Coordinate System transformations. The package infrastructure of TURBUSTAT relies heavily on the astropy testing and documentation infrastructure. ${ }^{21}$ The scikit-image ${ }^{22}$ (van der Walt et al. 2014) is used for morphological operations, contour finding, and fitting elliptical models. The scikit-learn ${ }^{23}$ (Pedregosa et al. 2011) provides a parallelized routine for calculating pairs of distances between data sets, which is required for the Cramer distance metric (Yeremi et al. 2014; Koch et al. 2017; Section 3.3). Most of the fitting routines in TURBUSTAT rely on the statsmodels package ${ }^{24}$ (Seabold \& Perktold 2010), which mimics the linear model fitting in the R programming language. ${ }^{25} \mathrm{We}$ also use statsmodels for the maximum-likelihood estimation for fitting PDFs. The astrodendro ${ }^{26}$ package creates a dendrogram to explore hierarchical structure (Rosolowsky et al. 2008; Goodman et al. 2009); TURBUSTAT creates statistical descriptions of the dendrogram.

TURBUSTAT also has a few optional dependencies. The spectral-cube ${ }^{27}$ (Ginsburg et al. 2019) and radio-beam ${ }^{28}$ packages provide convenient methods for handling large data sets and beam manipulation, respectively. The emcee ${ }^{29}$ (Foreman-Mackey et al. 2013) package provides an optional Markov chain Monte Carlo (MCMC) fitting for the size-line width relation in a PCA and distribution fitting for PDFs. The corner package ${ }^{30}$ (ForemanMackey 2016) creates convenient summary plots of the emcee sampler. For taking the fast-Fourier transform (FFT) of large data, the FFTW library ${ }^{31}$ (Frigo \& Johnson 2005) through the pyFFTW wrapper $^{32}$ (Gomersall 2016) can calculate the FFT in parallel.

\subsection{Method Implementation}

The 14 turbulence methods implemented in TURBUSTAT are implemented using a common framework to facilitate ease of

\footnotetext{
17 numpy.org

18 scipy.org

19 matplotlib.org

20 astropy.org/

21 github.com/astropy/astropy-helpers

22 scikit-image.org

23 scikit-image.org

24 statsmodels.org

25 r-project.org

26 dendrograms.readthedocs.io

27 spectral-cube.readthedocs.io

28 radio-beam.readthedocs.io

$29 \mathrm{dfm} . \mathrm{io} / \mathrm{emcee}$

30 github.com/dfm/corner.py

31 fftw.org

32 hgomersall.github.io/pyFFTW
}

use (the methods are presented in Section 2). Each method is implemented as a python class with a common set of steps.

1. Input. The data, FITS header, and other relevant input information (i.e., beam size, distance to region) are given as inputs when initializing the method class.

2. Calculation. Functions are defined in the class that performs the analysis. Depending on the complexity of the method, the computing steps are split into multiple parts. For example, the SPS has separate steps to (i) compute the power spectrum, (ii) fit the 1D power spectrum, (iii) fit the 2D power spectrum, and (iv) produce a summary plot and print fit statistics. Running each of these functions in the order given will compute the entire method. This step-bystep method allows for maximum user input when computing the method as the arguments and keyword arguments of each function can be altered.

3. All-in-one. The multistep approach described above can be cumbersome and requires remembering each step for computing the method. For ease of use, each method includes a run function that runs all of the steps with sensible default settings and optionally returns a figure summarizing the results. This approach is ideal for quickly computing a method for exploratory analyses. Most key settings can be altered in the run function so that users need only use this function for most cases.

4. Plotting and Summary. Each method has a separate function that returns a summary plot of the method, including fits to the outputs of the method.

Some methods have additional functions defined that return useful information about the method or data. For example, after computing the PDF of a data set, the PDF class has functions for returning the percentile of a given value in the data.

\subsection{Distance Metrics}

TURBUSTAT includes distance metrics that use an output of a method to compare two data sets. The methods implemented in TURBUSTAT measure properties of the underlying physics in observational data; these distance metrics are one approach for quantifying the difference in their physical properties. In previous works, we have used these distance metrics to find which methods are sensitive to different input parameters in sets of simulations (Yeremi et al. 2014; Boyden et al. 2016, 2018; Koch et al. 2017). We refer readers to Koch et al. (2017) for a full description of the distance metrics. The TURBUSTAT documentation also includes tutorials on the use of the distance metrics.

\subsection{Data Structure and Utilities}

Data Structure. TURBUSTAT is primarily intended to work with observational data products, namely $2 \mathrm{D}$ images and spectral-line data cubes. However, our goal is also to ensure that these methods are easily used with a wide array of data. As such, the TURBUSTAT methods accept two main input types: (1) utilizing the astropy I/O interface, in which the methods in TURBUSTAT expect a FITS Header Data Unit (HDU) as an input; (2) data can also be passed as a numpy array when converting to the FITS format is prohibitive. These inputs still require a FITS header be provided (see below). Data types from the spectral-cube package may also be used. The FITS header is required by most TURBUSTAT methods for converting pixel 
scales into sky coordinates or the spectral dimension in a data cube.

Generating FITS Headers. Non-observational data, such as simulated observations, may not be saved with mock WCS information included. For these cases, TURBUSTAT includes utility functions to generate a FITS header or HDU for the data.

Spectral Moments. Some methods in TURBUSTAT require a data cube while others need a 2D image representing some property of the data (i.e., integrated intensity or column density). TURBUSTAT includes utilities for calculating 2D moment images from a data cube-namely the zeroth (integrated intensity), first (centroid), and second (line width) moments-using the spectral-cube package, and saving the images as FITS files. Uncertainty maps for the moments can also be calculated, which are useful for down-weighting noisy regions in some methods.

\subsection{Generating Fractional Brownian Motion Images and Data Cubes}

Fractional Brownian motion (fBM) fields are useful, highly simplified versions of a turbulent field. They are created by setting the power-law amplitude and randomly drawing phases in the Fourier domain before transforming to real space. TURBUSTAT includes routines for creating 2D or 3D fBM fields and routines for creating mock optically thin $\mathrm{H}$ I data cubes given a 3D density and velocity field.

Two-dimensional fBM images can be created with a given power-law index, ellipticity (anisotropy), and angle of ellipticity. These routines are primarily used in TURBUSTAT to test methods but also provide useful examples (Section 4.2).

Three-dimensional fBM fields can also be generated, though the current implementation does not include the option for creating anisotropic fields.

Utilizing the aforementioned 3D fields, TURBUSTAT can generate mock optically thin $\mathrm{H}$ I data cubes from a given set of density and velocity fields. These mock data cubes are another useful tool for testing, and an example is shown in Section 4.1.

\subsection{Additional Features}

TURBUSTAT has a number of additional features that we briefly list here. We note that the use of these features is well described in the documentation with complete examples.

1. Beam Corrections. Most astronomical imaging, particularly in the radio and submillimeter, are oversampled relative to the area of a resolution element or the beam size. This leads to systematic correlations on scales of the order the beam size or smaller that affects most turbulence methods that utilize spatial information. For example, the spatial power spectra will steepen on scales similar to the beam size, approaching the power spectrum of the beam. TURBUSTAT includes routines for correcting the beam response in power-spectrum methods (SPS, MVC, VCA) that rely on the radio-beam package. The PCA implementation includes the beam correction described in Brunt \& Heyer (2002a). For other spatial methods where the transform remains in real space (wavelet, delta variance, statistical moments, SCF), we recommend closely examining the response on scales small or near the beam size, and possibly avoiding those region when fitting.
2. Apodizing kernels. The power-spectrum methods (SPS, MVC, VCA) use an FFT that will exhibit the Gibbs phenomenon (ringing) when there is signal at the edge of the image. The edges of an image can be tapered to avoid this ringing using an apodizing kernel. TURBUSTAT includes several options, with a tutorial demonstrating their effect on the power spectrum, based on the routines implemented in photutils ${ }^{33}$ (Bradley et al. 2019).

3. Parallelized FFTs. Computing the FFT is a bottleneck when running FFT-based methods (SPS, MVC, VCA, wavelets, delta variance) on large data sets. To speed the FFT up, pyFFTW ${ }^{34}$ (Gomersall 2016) can optionally be used to run the FFT in parallel.

4. Segmented Linear Model. TURBUSTAT implements a segmented linear model described in Muggeo (2003) that fits for a piece-wise linear model and the position of the breaks. This model is used by default for the VCS, and can be optionally used for all power-spectrum-based methods. For example, this model can be used to constrain break points in spatial power spectra that are related to a galaxy's disk scale height (e.g., Combes et al. 2012).

5. 2D Elliptical Power-law Model. TURBUSTAT includes an elliptical power-law model, adapted from Tessore \& Metcalf (2015; their Equations (3)-(5)), for fitting 2D power spectra and constraining anisotropy. The model is defined by three parameters: the power-law index, the ellipticity, and the elliptical angle. Ellipticity parameterizes anisotropy and is defined such that 0 is infinitely anisotropic and 1 is isotropic. The elliptical angle is the angle between the $x$-axis and the direction of the anisotropy. An example of this model is shown in Section 4.2.2. The TURBUSTAT implementation fits the logarithm of the 2D power spectrum and estimates uncertainties using residual bootstrapping.

\section{TURBUStat Examples}

This section presents examples of the methods in TURBUSTAT with idealized synthetic observations (Section 3.5). We highlight the code's ability to recover expected parameters for methods that utilize data cubes (Section 4.1) and 2D images (Section 4.2). Scripts to reproduce these examples are available at https:// github.com/Astroua/TurbuStat/tree/master/Examples.

\subsection{Examples with Position-Position-Velocity Cubes \\ 4.1.1. Example Cubes}

For the following examples, we generate four idealized spectral-line data cubes. These cubes are generated from 3D fBM fields with a shape of $256^{3}$ and an index of -4 for the density and velocity fields. We assume an isotropic velocity and so only generate one component of the velocity. The velocity field has a dispersion of $10 \mathrm{~km} \mathrm{~s}^{-1}$, and the density field has a dispersion of $1 \mathrm{~cm}^{-3}$. The set of four fields differ only in the random seed used to generate their phases.

The density fBM field is not positive definite and must be altered to ensure that it is. Many approaches have been proposed to create a positive definite field from a fBM field, including taking

\footnotetext{
33 photutils.readthedocs.io

34 hgomersall.github.io/pyFFTW
} 


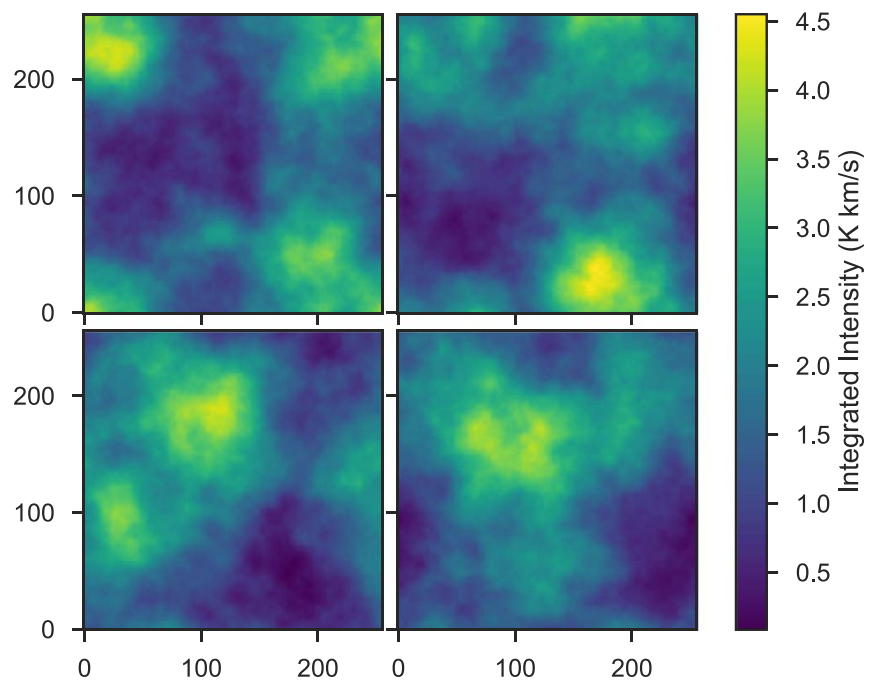

Figure 1. Integrated intensity maps of the idealized fBM H I cubes used in Section 4.1. We generated the cubes from density and velocity fields with indices of -4 that differ only in their random phases.

the absolute value (Stutzki et al. 1998), taking the exponential to generate a log-normal distribution (Brunt \& Heyer 2002a; Ossenkopf et al. 2006; Roman-Duval et al. 2011), and subtracting the minimum value of the field (Miville-Deschênes et al. 2003) or some multiple of the field's standard deviation (Ossenkopf et al. 2006). We mimic the latter approach by adding the standard deviation of the density field to itself and setting values that remain negative to zero. This distorts the index of the density field's power-spectrum index, however, the measured indices vary by less than 0.1 from the original index of -4 .

We then generate four spectral-line data cubes of $\mathrm{HI}$ emission assuming optically thin conditions and a temperature of $100 \mathrm{~K}$ (the thermal velocity dispersion $\sigma_{\text {therm }}=0.76 \mathrm{~km} \mathrm{~s}^{-1}$ ). We set the velocity channel width to be $0.2 \mathrm{~km} \mathrm{~s}^{-1}$, and thus expect the spectral line profiles to be smooth. Adopting a thermal line width is necessary for smoothing away shot noise on small scales (Lazarian et al. 2001). Figure 1 shows the integrated intensity images of the four cubes.

We note that these are small cubes that have been generated for these examples. We have chosen to do this to make reproducing these examples computationally inexpensive. However, the choice to use small cubes does impose limitations. The aforementioned shot noise that arises from having a finite number of emitters along the line of sight is a significant problem here (Lazarian et al. 2001). Esquivel et al. (2003) and Chepurnov \& Lazarian (2009) demonstrate that this numerical effect drastically affects the recovered indices for the VCA and VCS. By selecting a steep field index (-4) and by smoothing the data cubes with the thermal line width $\left(\sigma_{\text {therm }}=0.76 \mathrm{~km} \mathrm{~s}^{-1}\right)$, we ensure that velocity slices remain close to the thin VCA regime on scales larger than 2 pixels (based on Equations (12)-14 from Esquivel et al. 2003).

We also note that these idealized data cubes have no density-velocity correlations (Esquivel et al. 2007) and are not affected by absorption or optical-depth effects (Lazarian \& Pogosyan 2004; Burkhart et al. 2013a).

\subsection{2. $V C A$}

Lazarian \& Pogosyan (2000) predict that the SPS index will change as a function of the velocity channel width due to

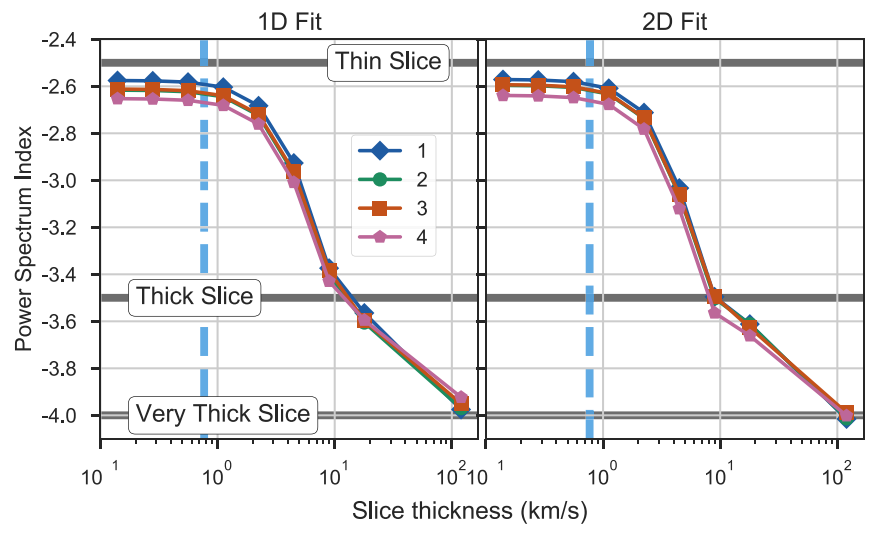

Figure 2. Measured VCA index from fits to the 1D (left) and 2D power spectra (right) as a function of velocity slice thickness for four example cubes generated with different random seeds (Section 4.1.1). The vertical line is the thermal velocity dispersion used for smoothing, and the horizontal lines are the predicted slopes in the thin $(-2.5)$, thick $(-3.5)$, and very thick velocity $(-4.0)$ regimes for density and velocity fields with an index of -4 . Shot noise and smoothing with the thermal velocity dispersion result in the thin velocity regime not being recovered (Esquivel et al. 2003; Chepurnov \& Lazarian 2009). Indices fitted to the $2 \mathrm{D}$ power spectrum are moderately steeper than the $1 \mathrm{D}$ power-spectrum indices due to the larger number of samples on small scales (large frequencies) in the 2D power spectrum. The TURBUSTAT implementation of the VCA and spatial power spectrum recover the expected indices.

differences in the influence of the underlying velocity and density fields. As the channel width increases, velocity fluctuations are averaged out and the power-spectrum index approaches the index of the density field. We have chosen the VCA as an example because the analytic relation for the index with spectral channel width provides a good test case to ensure that the TURBUSTAT implementation, and the mock data cube creation, are correct.

There are three regimes for the VCA index described by Lazarian \& Pogosyan (2000), (1) the thin velocity regime dominated by velocity fluctuations: $m=-3-\left(\gamma_{v}+3\right) / 2$, where $m$ is the power-spectrum index and $\gamma_{v}$ is the velocity field index; (2) the thick velocity regime where most velocity fluctuations have been averaged over: $m=-3+\left(\gamma_{v}+3\right) / 2$; and (3) the very thick velocity regime where all velocity channels have been integrated over: $m=\gamma_{n}$, where $\gamma_{n}$ is the density field index. Note that these relations are for the steep density regime $\left(\gamma_{n}<-3\right)$; see Lazarian \& Pogosyan (2000) for the shallow density regime. For the $\gamma_{v}=\gamma_{n}=-4$ for these examples, we expect $m_{\text {thin }}=-2.5, \quad m_{\text {thick }}=-3.5$, and $m_{\text {verythick }}=-4$, which are shown as horizontal lines in Figure 2.

Using TURBUSTAT's VCA and SPS implementations, we fit $1 \mathrm{D}$ and $2 \mathrm{D}$ power-law models to the power spectra as a function of channel width. Figure 2 shows the recovered indices of the four example cubes. For thin velocity channels, the recovered indices are $\sim-2.6$ and do not approach the expected -2.5 in the thin regime due to shot noise and smoothing from the thermal line width, shown with the blue vertical line. For larger channel widths, we recover the expected indices in the thick and very thick regimes. The $2 \mathrm{D}$ power-law indices are moderately steeper in the thick velocity regime as these fits are dominated by the larger number of samples at large spatial frequencies. The variation in indices between the four cubes is small $(<0.1)$.

\subsection{3. $P C A$}

We also demonstrate our implementation of PCA on the four example cubes. Adopting a minimum eigenvalue of 0.001 to 


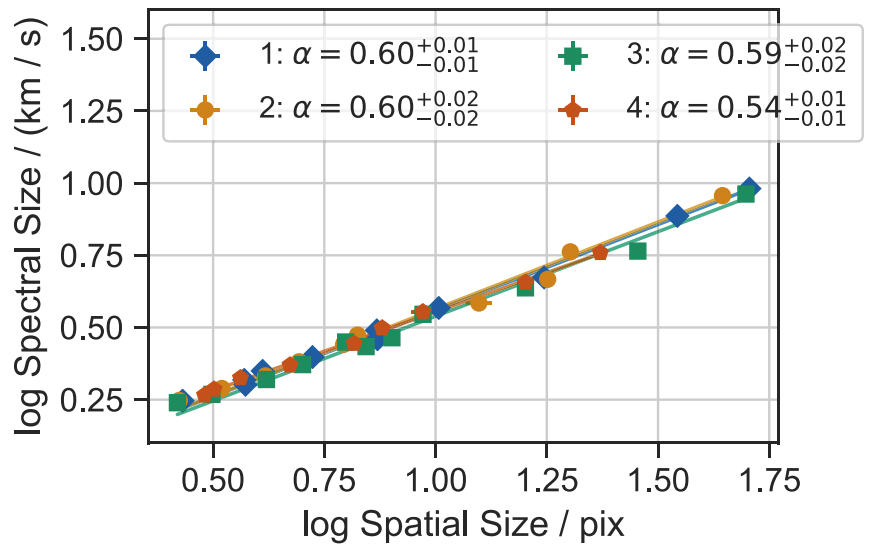

Figure 3. Size-line width relations from PCA for the four example cubes. The expected index of the size-line width relation for a velocity field with an index of -4 is $1 / 2$; the fitted indices are consistently steeper than the expected value. These results demonstrate that similar slopes are consistently recovered with the TURBUSTAT PCA implementation.

avoid numerical noise in the PCA decomposition, we calculate the spatial and spectral scales and fit the size-line width relation for the four cubes, shown in Figure 3. For this example, we fit the size-line width relations with orthogonal distance regression. ${ }^{35}$

For a velocity field index of -4 , we expect the size-line width relation to have an index of 0.5 . The recovered indices are consistently higher than 0.5 , which we expect is due to a small number of eigenvalues containing useful information, and three of the cubes have indices consistent within the uncertainty. The fourth cube has a smaller index-closer to the expected indexthan the other cubes which may be due to the autocorrelation surface of the first eigenimage not containing the $1 / e$ contour used to find the spatial scale. This issue has been noted in other PCA works (e.g., Roman-Duval et al. 2011). We also note that no correction factor has been used here, as is typically done to relate the measured index to the index of the energy spectrum (Brunt \& Heyer 2002a; Roman-Duval et al. 2011). The aforementioned works impose a log-normal distribution on the density fBM field, which is not done here, and the applicability of these correction factors may not be suitable for this case. However, TURBUSTAT includes the option to apply the correction factor from Brunt \& Heyer (2002a).

\subsection{Examples with $2 D$ Images}

We demonstrate some of the methods that apply to $2 \mathrm{D}$ images in this section, namely the recovery of known indices from fBM images and fitting elliptical power-law models to constrain anisotropy.

\subsubsection{Image Index Recovery}

We generate a series of 2D fBM images with indices ranging from $\beta=0.5-4$ and different random seeds to demonstrate how well the delta variance, SPS, and wavelets recover the indices. Each of these methods provides complementary information based on their fitted slopes. The delta variance and wavelet transforms are similar to a second-order structure function and their measured slopes are related to the powerspectrum index with $\beta=m_{\text {delta-variance }}+2$ and $\beta=\left(m_{\text {wavelet }}+\right.$ 1)/2, respectively.

\footnotetext{
35 Implemented in scipy.
}

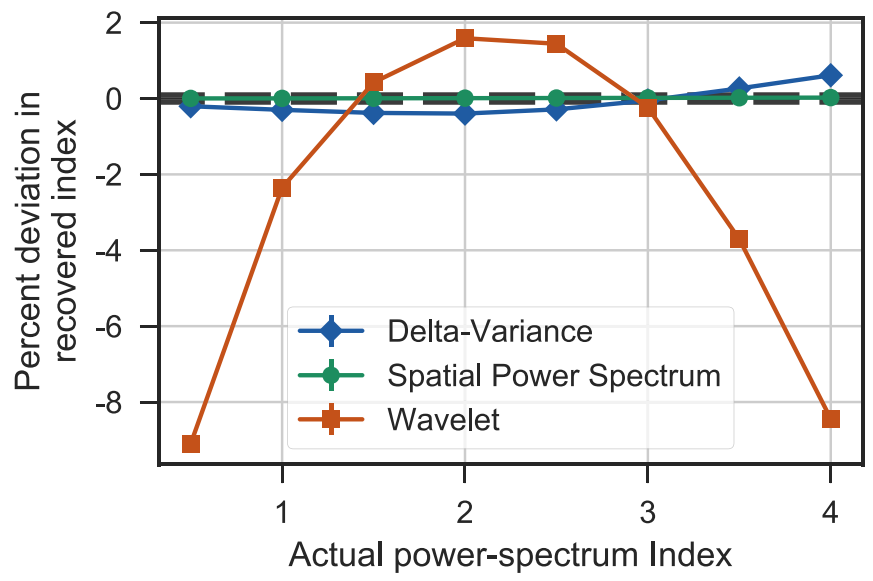

Figure 4. Percent deviation between the measured and actual power-spectrum index for fBM images with the delta variance (blue diamonds), spatial powerspectrum (green circles), and wavelet (orange squares) methods. The former two methods accurately recover the power-spectrum index to within a few percent, which for observational data will likely be smaller than the uncertainty. The wavelet method shows larger deviations that depend strongly on the index of the image. Over the range of indices expected for interstellar turbulence, the delta variance and spatial power spectrum accurately recover the index of an image.

Figure 4 shows the percent deviation of the measured index from the actual index across the range of fBM images. The SPS recovers the correct index in each case, while the index from the delta variance deviates by $<1 \%$. The index from the wavelet transform, however, has larger systematic deviations that vary across the range of fBM image indices shown. These deviations suggest that the delta variance and SPS provide more accurate results than the wavelet transform.

\subsubsection{Modeling Spatial Anisotropy}

There is significant interest in studying the connection between intensity and velocity anisotropy with magnetic field structure in the ISM (e.g., Esquivel \& Lazarian 2005; Burkhart et al. 2014; Kalberla \& Kerp 2016; Kandel et al. 2017). In this example, we demonstrate TURBUSTAT's implementation of a 2D elliptical power-law model for fitting power spectra and other 2D surfaces. The model is described in Section 3.6.

The left panel of Figure 5 shows a fBM field with a slope of -3 and ellipticity of $0.4^{36}$ oriented $60^{\circ}$ above the $x$-axis in the figure. Using the SPS, we find the $2 \mathrm{D}$ power spectrum shown in the right panel, which highlights the anisotropy of the structure. Contours of the fitted elliptical power law are shown with solid lines in the figure. The model correctly recovers the aforementioned fBM image parameters.

This model can be used with other power-spectrum-based methods in TURBUSTAT, including MVC and VCA, and the SCF correlation surface to constrain anisotropy in images.

\section{Conclusions}

We introduce TURBUSTAT, a Python package for turbulence statistics. Currently, the package includes 14 methods presented in the literature for recovering turbulent properties from ISM observations. This paper describes the capabilities of the implementations and other utilities in the package. We also present a few examples (Section 4) that demonstrate the code's ability to recover the expected properties. We note that

\footnotetext{
${ }^{36}$ Defined such that an ellipticity of 1.0 is isotropic and 0.0 is infinitely anisotropic.
} 


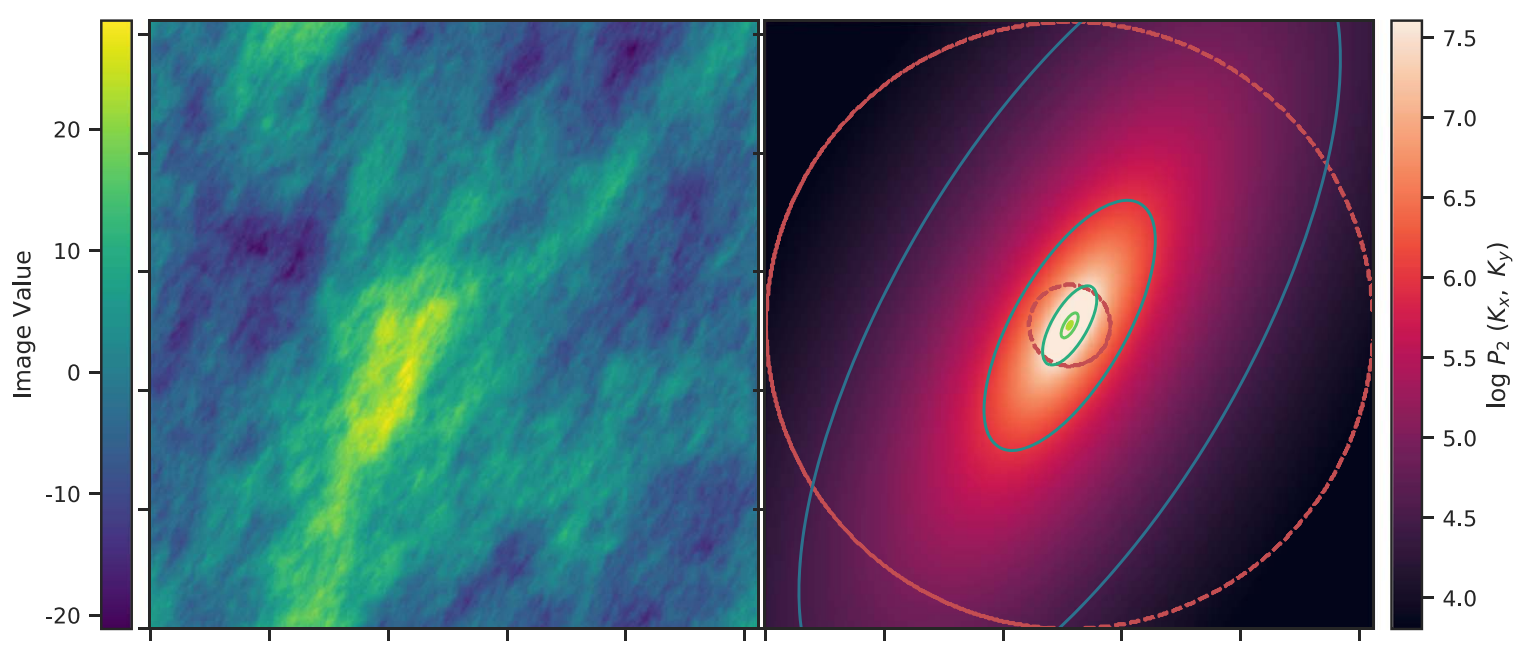

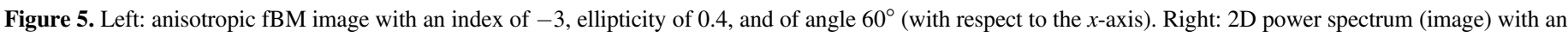

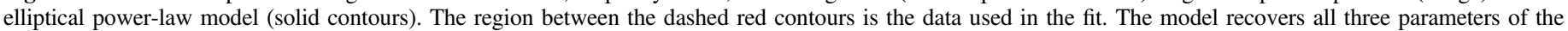
anisotropic $\mathrm{fBM}$ image to within the fit uncertainties.

additional turbulence data sets in FITS format can be obtained online as part of the Catalog for Astrophysical Turbulence Simulations (CATS) project. $^{37}$ CATS also includes spectral line data compatible with TURBUSTAT.

While this version of TURBUSTAT includes many methods for recovering turbulent properties, there are a number of other methods that we hope to include in the future. Some examples include: structure functions (e.g., Boldyrev et al. 2002; Padoan et al. 2003), the phase coherence index (Burkhart \& Lazarian 2016), the brightness distribution index (Sawada et al. 2012), the velocity gradient technique (e.g., Yuen \& Lazarian 2017; Lazarian et al. 2018), wavelet-based crosscorrelation analysis (Arshakian \& Ossenkopf 2016), and complete VCS modeling (Chepurnov et al. 2010, 2015).

TURBUSTAT is an open source ${ }^{38}$ and has thorough documentation and tutorials available. ${ }^{39}$ We welcome feedback, recommendations, and contributions from the community to improve the ongoing development of TURBUSTAT.

We thank Caleb Ward for important contributions to the code in its early development. We are grateful for feedback and issues reported by Dario Colombo, Jesse Feddersen, Simon Glover, Jonathan Henshaw, Andrés Izquierdo, and Sac Medina. We thank the anonymous referee for the careful reading of the manuscript and comments. E.W.K. is supported by a Postgraduate Scholarship from the Natural Sciences and Engineering Research Council of Canada (NSERC). E.W.K. and E.W.R. are supported by a Discovery Grant from NSERC (RGPIN-2012-355247; RGPIN-2017-03987). This research was enabled in part by support provided by WestGrid, ${ }^{40}$ Compute Canada, ${ }^{41}$ and CANFAR. ${ }^{42}$

Software: astropy (Astropy Collaboration et al. 2018), matplotlib (Hunter 2007), seaborn (Waskom et al. 2017), numpy (Oliphant 2006), scipy (Jones et al. 2001), scikit-image (van der Walt et al.

\footnotetext{
37 www.mhdturbulence.com

38 github.com/Astroua/TurbuStat

39 turbustat.readthedocs.io

40 www.westgrid.ca

41 www.computecanada.ca

42 www.canfar.net
}

2014), scikit-learn (Pedregosa et al. 2011), statsmodels (Seabold \& Perktold 2010), astrodendro (dendrograms.readthedocs.io), spectral-cube (spectral-cube.readthedocs.io), radio-beam (radio-beam. readthedocs.io), emcee (Foreman-Mackey et al. 2013), corner (Foreman-Mackey 2016), pyFFTW (Gomersall 2016) and FFTW (Frigo \& Johnson 2005)

\section{Appendix A \\ Issues with Wavelet Normalization}

When implementing the wavelet transform from Gill \& Henriksen (1990), we found that similar quantitative results from Gill \& Henriksen (1990) could only be reproduced using a Mexican hat kernel that was not normalized. With a normalized kernel, the slope is decreased by 2 compared to the slopes reported by Gill \& Henriksen (1990). This difference is due to the Mexican hat wavelet's close relation to the Laplacian of a Gaussian kernel. The Gaussian kernel carries units of length ${ }^{-2}$ and its response remains constant across all scales (Lindeburg 1994). Each derivative causes the response to decay by roughly $1 / \sigma$, since the derivative effectively introduces an additional unit of length ${ }^{-1}$. To correct for the decaying response, the convolution of the image and Mexican hat wavelet should be multiplied with $\sigma^{2}$, restoring the length ${ }^{-2}$ units of the Gaussian kernel. This normalization accounts for the difference of 2 we find in the slopes. This approach is known as scale-normalized derivatives and is essential in blob detection algorithms (Lindeburg 1994).

While the wavelet normalization does not change the qualitative results and interpretation from Gill \& Henriksen (1990), deviations from a power-law relation can be hidden when an unnormalized kernel is used. Figure 6 shows the wavelet transform for the same data set with and without normalization (see also Figure 12 in Boyden et al. 2016). The unnormalized and normalized transforms have slopes of $2.50 \pm 0.01$ and $0.50 \pm 0.01$, respectively, when fitting on scales from 2.5 to 40 pixels. Of note is the larger deviations in the normalized transform compared to the unnormalized transform on scales larger than 40 pixels.

Our implementation of the wavelet transform in the TURBUSTAT package allows for the normalization to be 


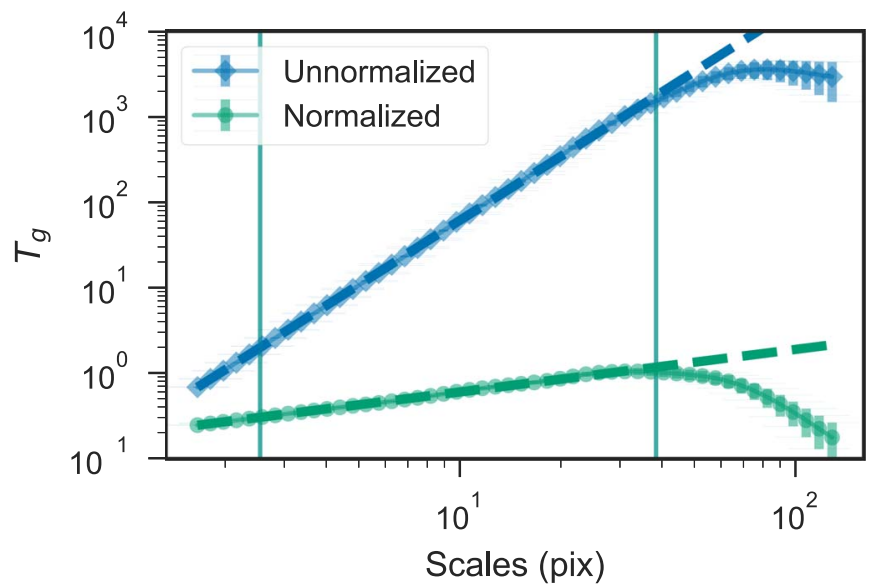

Figure 6. Wavelet transform with (green circles) and without (blue diamonds) a normalized Mexican Hat wavelet. The dashed lines are the fits to the respective curves, and the region used for the fit is indicated with the vertical lines. Deviations from a single power-law relation are more significant in the normalized transform.

disabled to so the Gill \& Henriksen (1990) results can be reproduced, though a warning is printed when doing so.

\section{Appendix B}

\section{Comparison to the Delta Variance IDL Code}

The original version of the delta variance described in Ossenkopf et al. (2008a, 2008b) is publicly available. ${ }^{43}$ Figure 7 shows the delta-variance curve from an fBM image with an index of -3.0 , as used in Section 4.2. The figures shows that the TURBUSTAT implementation recovers a deltavariance curve whose slope is consistent with the IDL version, within uncertainty. There is an offset between the curves that results from differences in the implementations of the convolution step. ${ }^{44}$ The offset is constant within uncertainty for all scales and remains the same when tested on fBM images with different indices. Thus, the offset does not affect the recovered slope.

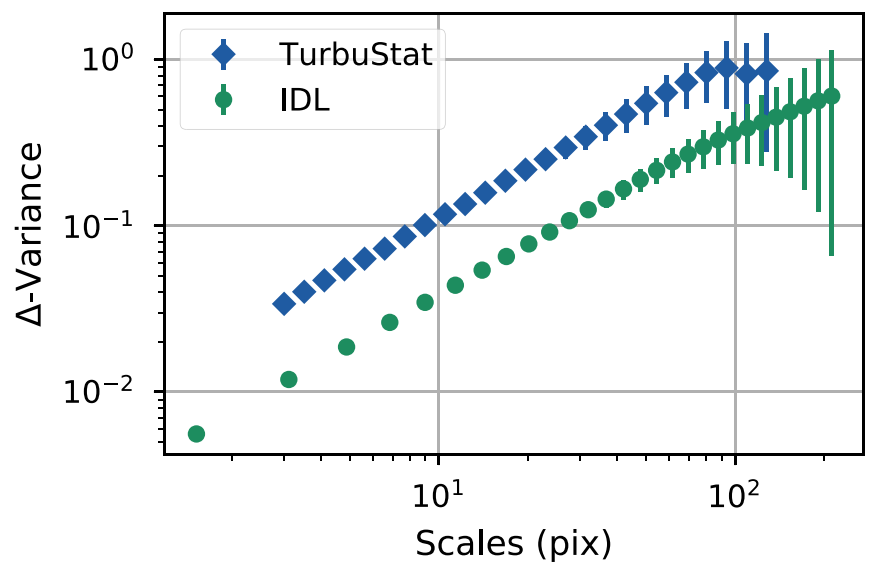

Figure 7. Delta-variance curves from TURBUSTAT (blue diamonds) and the original IDL implementation described in Ossenkopf et al. (2008a; green circles) of an fBM image with an index of -3.0 . The recovered slope of both curves is within uncertainty of the expected slope of 1.0. The offset between the curves results from differences in the implementation of the convolution step.

\section{Appendix C Scaling Tests}

We summarize a set of performance tests in this section for the 14 statistical methods in TurbuStat. These tests were run with the default settings on synthetic data ranging from sizes of $256^{2}$ to $2048^{2}$ for $2 \mathrm{D}$ images and $256^{3}$ to $2048^{3}$ for spectralline data cubes. We ran the tests on a compute node with two Intel E5-2683 v4 Broadwell processors and $512 \mathrm{~GB}^{45}$; the times are based on running on a single processor.

Figure 8 summarizes these tests by showing the five methods with the highest memory usage or run time. We note that the memory tests do not include the memory usage of the data products. Machine-readable tables are available for all methods as online material.

Scripts to reproduce these scaling tests are available at https:// github.com/Astroua/TurbuStat/tree/master/Examples.

\footnotetext{
43 hera.ph1.uni-koeln.de/ ossk/Myself/deltavariance.html

44 TURBUSTAT uses astropy's FFT convolution function; docs.astropy.org/ en/stable/convolution.
}

\footnotetext{
$\overline{45}$ This is a large $512 \mathrm{G}$ on the cedar cluster; docs.computecanada.ca/wiki/ Cedar.
} 


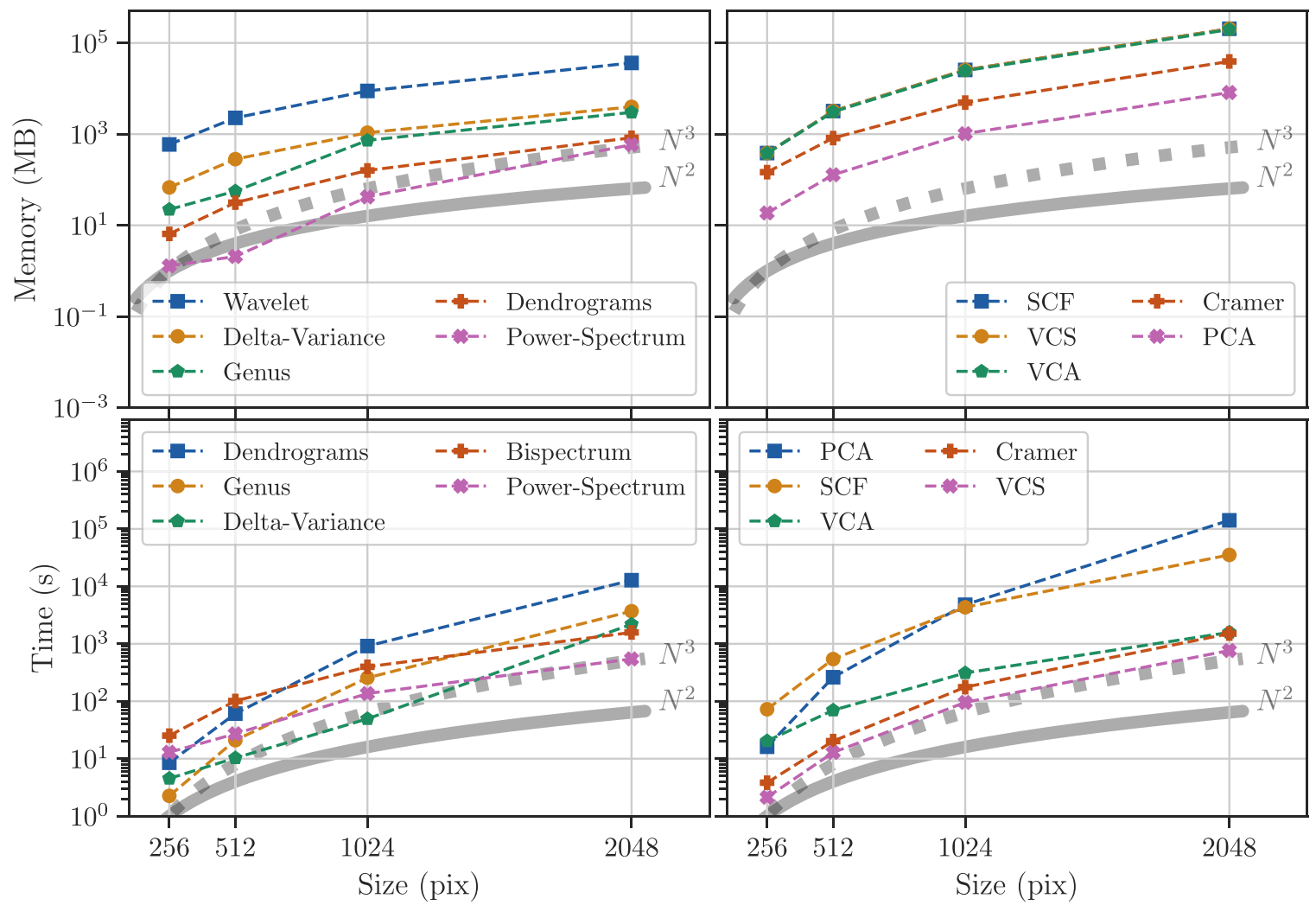

Figure 8. Memory (top row) and run time (bottom row) tests for 2D (left column) and 3D (right column) statistical methods as a function of image/cube size in pixels. The solid and dash gray lines indicate $N^{2}$ and $N^{3}$ scaling relations for image/cube size $N$. Each panel shows the five methods ordered from most to least usage in each panel, with the order shown in the legends. Other 2D methods use less memory or have a shorter run time than those plotted. The data used to create this figure are available.

\section{ORCID iDs}

Eric W. Koch ๑ https://orcid.org/0000-0001-9605-780X

Erik W. Rosolowsky (1) https://orcid.org/0000-0002-

5204-2259

Ryan D. Boyden (1) https://orcid.org/0000-0001-9857-1853

Adam Ginsburg (1) https://orcid.org/0000-0001-6431-9633

Stella S. R. Offner (1) https://orcid.org/0000-0003-1252-9916

\section{References}

Armstrong, J. W., Rickett, B. J., \& Spangler, S. R. 1995, ApJ, 443, 209 Arshakian, T. G., \& Ossenkopf, V. 2016, A\&A, 585, A98

Astropy Collaboration, Price-Whelan, A. M., Sipő́cz, B. M., et al. 2018, AJ, 156,123

Beaumont, C. N., Offner, S. S. R., Shetty, R., Glover, S. C. O., \& Goodman, A. A. 2013, ApJ, 777, 173

Bensch, F., Stutzki, J., \& Ossenkopf, V. 2001, A\&A, 366, 636

Bialy, S., Burkhart, B., \& Sternberg, A. 2017, ApJ, 843, 92

Boldyrev, S., Nordlund, Å., \& Padoan, P. 2002, ApJ, 573, 678

Boyden, R. D., Koch, E. W., Rosolowsky, E. W., \& Offner, S. S. R. 2016, ApJ, 833, 233

Boyden, R. D., Offner, S. S. R., Koch, E. W., \& Rosolowsky, E. W. 2018, ApJ, 860, 157

Bradley, L., Sipocz, B., Robitaille, T., et al. 2019, astropy/photutils: v0.6, Zenodo, doi:https://doi.org/10.5281/zenodo.2533376

Brunt, C. M., \& Heyer, M. H. 2002a, ApJ, 566, 276

Brunt, C. M., \& Heyer, M. H. 2002b, ApJ, 566, 289

Brunt, C. M., \& Heyer, M. H. 2013, MNRAS, 433, 117

Burkhart, B. 2018, ApJ, 863, 118

Burkhart, B., Collins, D. C., \& Lazarian, A. 2015a, ApJ, 808, 48

Burkhart, B., Falceta-Gonçalves, D., Kowal, G., \& Lazarian, A. 2009, ApJ, 693, 250

Burkhart, B., \& Lazarian, A. 2016, ApJ, 827, 26
Burkhart, B., Lazarian, A., Balsara, D., Meyer, C., \& Cho, J. 2015b, ApJ, 805,118

Burkhart, B., Lazarian, A., \& Gaensler, B. M. 2012, ApJ, 749, 145

Burkhart, B., Lazarian, A., Goodman, A., \& Rosolowsky, E. 2013, ApJ, 770,141

Burkhart, B., Lazarian, A., Leão, I. C., de Medeiros, J. R., \& Esquivel, A. 2014, ApJ, 790, 130

Burkhart, B., Lazarian, A., Ossenkopf, V., \& Stutzki, J. 2013a, ApJ, 771, 123

Burkhart, B., Ossenkopf, V., Lazarian, A., \& Stutzki, J. 2013b, ApJ, 771, 122

Burkhart, B., Stalpes, K., \& Collins, D. C. 2017, ApJL, 834, L1

Burkhart, B., Stanimirović, S., Lazarian, A., \& Kowal, G. 2010, ApJ, 708, 1204

Chen, H. H.-H., Burkhart, B., Goodman, A., \& Collins, D. C. 2018, ApJ, 859,162

Chepurnov, A., Burkhart, B., Lazarian, A., \& Stanimirovic, S. 2015, ApJ, 810,33

Chepurnov, A., Gordon, J., Lazarian, A., \& Stanimirović, S. 2008, ApJ, 688, 1021

Chepurnov, A., \& Lazarian, A. 2009, ApJ, 693, 1074

Chepurnov, A., \& Lazarian, A. 2010, ApJ, 710, 853

Chepurnov, A., Lazarian, A., Stanimirović, S., Heiles, C., \& Peek, J. E. G. 2010, ApJ, 714, 1398

Cho, J., \& Lazarian, A. 2003, MNRAS, 345, 325

Collins, D. C., Kritsuk, A. G., Padoan, P., et al. 2012, ApJ, 750, 13

Combes, F., Boquien, M., Kramer, C., et al. 2012, A\&A, 539, A67

Correia, C., Lazarian, A., Burkhart, B., Pogosyan, D., \& De Medeiros, J. R. 2016, ApJ, 818, 118

Crovisier, J., \& Dickey, J. M. 1983, A\&A, 122, 282

Elmegreen, B. G., \& Scalo, J. 2004, ARA\&A, 42, 211

Esquivel, A., \& Lazarian, A. 2005, ApJ, 631, 320

Esquivel, A., \& Lazarian, A. 2010, ApJ, 710, 125

Esquivel, A., Lazarian, A., Horibe, S., et al. 2007, MNRAS, 381, 1733

Esquivel, A., Lazarian, A., Pogosyan, D., \& Cho, J. 2003, MNRAS, 342, 325

Federrath, C., \& Klessen, R. S. 2012, ApJ, 761, 156

Federrath, C., Klessen, R. S., \& Schmidt, W. 2008, ApJL, 688, L79

Foreman-Mackey, D. 2016, JOSS, 1, 24 
Foreman-Mackey, D., Hogg, D. W., Lang, D., \& Goodman, J. 2013, PASP, 125,306

Frigo, M., \& Johnson, S. G. 2005, Proc. IEEE, 93, 216

Gaches, B. A. L., Offner, S. S. R., Rosolowsky, E. W., \& Bisbas, T. G. 2015, ApJ, 799, 235

Gaensler, B. M., Haverkorn, M., Burkhart, B., et al. 2011, Natur, 478, 214

Gill, A. G., \& Henriksen, R. N. 1990, ApJL, 365, L27

Ginsburg, A., Koch, E., Robitaille, T., et al. 2019, radio-astro-tools/spectralcube: v0.4.4, Zenodo, doi:https://doi.org/10.5281/zenodo.2573901

Goldreich, P., \& Sridhar, S. 1995, ApJ, 438, 763

Gomersall, H. 2016, pyFFTW, Zenodo, doi:https://doi.org/10.5281/zenodo. 59508

González-Casanova, D. F., \& Lazarian, A. 2017, ApJ, 835, 41

González-Casanova, D. F., Lazarian, A., \& Cho, J. 2018, MNRAS, 475, 3324

Goodman, A. A., Rosolowsky, E. W., Borkin, M. A., et al. 2009, Natur, 457, 63

Hagihira, S., Takashina, M., Mori, T., Mashimo, T., \& Yoshiya, I. 2001, Anesth. Analg., 93, 966

Haworth, T. J., Glover, S. C. O., Koepferl, C. M., Bisbas, T. G., \& Dale, J. E. 2018, NewAR, 82,

Herron, C. A., Burkhart, B., Gaensler, B. M., et al. 2018, ApJ, 855, 29

Heyer, M., Gong, H., Ostriker, E., \& Brunt, C. 2008, ApJ, 680, 420

Heyer, M. H., \& Schloerb, F. P. 1997, ApJ, 475, 173

Hill, A. S., Mac Low, M.-M., Gatto, A., \& Ibáñez-Mejía, J. C. 2018, ApJ, 862,55

Houlahan, P., \& Scalo, J. 1990, ApJS, 72, 133

Houlahan, P., \& Scalo, J. 1992, ApJ, 393, 172

Hull, C. L. H., Mocz, P., Burkhart, B., et al. 2017, ApJL, 842, L9

Hunter, J. D. 2007, CSE, 9, 90

Iacobelli, M., Burkhart, B., Haverkorn, M., et al. 2014, A\&A, 566, A5

Imara, N., \& Burkhart, B. 2016, ApJ, 829, 102

Jones, E., Oliphant, T., Peterson, P., et al. 2001, SciPy: Open source scientific tools for Python, http://www.scipy.org/

Kalberla, P. M. W., \& Kerp, J. 2016, A\&A, 595, A37

Kandel, D., Lazarian, A., \& Pogosyan, D. 2016, MNRAS, 461, 1227

Kandel, D., Lazarian, A., \& Pogosyan, D. 2017, MNRAS, 464, 3617

Koch, E., Ginsburg, A., Rosolowsky, E., Feddersen, J., \& Glover, S. 2019, Astroua/TurbuStat: First Major Release, Zenodo, doi:https://doi.org/10. 5281/zenodo. 2648076

Koch, E. W., Ward, C. G., Offner, S., Loeppky, J. L., \& Rosolowsky, E. W. 2017, MNRAS, 471, 1506

Kowal, G., Lazarian, A., \& Beresnyak, A. 2007, ApJ, 658, 423

Kritsuk, A. G., Norman, M. L., Padoan, P., \& Wagner, R. 2007, ApJ, 665, 416

Krumholz, M. R., Bate, M. R., Arce, H. G., et al. 2014, in Protostars and Planets VI, ed. H. Beuther et al. (Tucson, AZ: Univ. Arizona Press), 243

Krumholz, M. R., Burkhart, B., Forbes, J. C., \& Crocker, R. M. 2018, MNRAS, 477, 2716

Krumholz, M. R., McKee, C. F., \& Tumlinson, J. 2009, ApJ, 699, 850

Lazarian, A. 2009, SSRv, 143, 357

Lazarian, A., \& Esquivel, A. 2003, ApJL, 592, L37

Lazarian, A., \& Pogosyan, D. 2000, ApJ, 537, 720

Lazarian, A., \& Pogosyan, D. 2004, ApJ, 616, 943

Lazarian, A., \& Pogosyan, D. 2006, ApJ, 652, 1348

Lazarian, A., \& Pogosyan, D. 2008, ApJ, 686, 350

Lazarian, A., Pogosyan, D., \& Esquivel, A. 2002, in ASP Conf. Ser. 276, Seeing Through the Dust: The Detection of HI and the Exploration of the ISM in Galaxies, ed. A. R. Taylor, T. L. Landecker, \& A. G. Willis (San Francisco, CA: ASP), 182

Lazarian, A., Pogosyan, D., Vázquez-Semadeni, E., \& Pichardo, B. 2001, ApJ, 555,130
Lazarian, A., Yuen, K. H., Ho, K. W., et al. 2018, ApJ, 865, 46

Lindeburg, T. 1994, J. Appl. Stat., 21, 224

Lombardi, M., Alves, J., \& Lada, C. J. 2015, A\&A, 576, L1

Martin, P. G., Blagrave, K. P. M., Lockman, F. J., et al. 2015, ApJ, 809, 153

McKee, C. F., \& Ostriker, E. C. 2007, ARA\&A, 45, 565

Meyer, C. D., Balsara, D. S., Burkhart, B., \& Lazarian, A. 2014, MNRAS, 439, 2197

Miesch, M. S., \& Bally, J. 1994, ApJ, 429, 645

Miesch, M. S., \& Scalo, J. M. 1995, ApJL, 450, L27

Miville-Deschênes, M. A., Levrier, F., \& Falgarone, E. 2003, ApJ, 593, 831

Muggeo, V. M. 2003, Stat. Med., 22, 3055

Muller, E., Stanimirović, S., Rosolowsky, E., \& Staveley-Smith, L. 2004, ApJ, 616,845

Oliphant, T. 2006, NumPy: A guide to NumPy (USA: Trelgol Publishing)

Ossenkopf, V., Esquivel, A., Lazarian, A., \& Stutzki, J. 2006, A\&A, 452, 223

Ossenkopf, V., Klessen, R. S., \& Heitsch, F. 2001, A\&A, 379, 1005

Ossenkopf, V., Krips, M., \& Stutzki, J. 2008a, A\&A, 485, 917

Ossenkopf, V., Krips, M., \& Stutzki, J. 2008b, A\&A, 485, 719

Ostriker, E. C., McKee, C. F., \& Leroy, A. K. 2010, ApJ, 721, 975

Ostriker, E. C., Stone, J. M., \& Gammie, C. F. 2001, ApJ, 546, 980

Padoan, P., Bally, J., Billawala, Y., Juvela, M., \& Nordlund, A. 1999, ApJ, 525,318

Padoan, P., Boldyrev, S., Langer, W., \& Nordlund, Å. 2003, ApJ, 583, 308

Padoan, P., Goodman, A. A., \& Juvela, M. 2003, ApJ, 588, 881

Padoan, P., Juvela, M., Kritsuk, A., \& Norman, M. L. 2006, ApJL, 653, L125

Padoan, P., Rosolowsky, E. W., \& Goodman, A. A. 2001, ApJ, 547, 862

Pedregosa, F., Varoquaux, G., Gramfort, A., et al. 2011, Journal of Machine Learning Research, 12, 2825

Pingel, N. M., Lee, M.-Y., Burkhart, B., \& Stanimirović, S. 2018, ApJ, 856,136

Portillo, S. K. N., Slepian, Z., Burkhart, B., Kahraman, S., \& Finkbeiner, D. P. 2018, ApJ, 862, 119

Roman-Duval, J., Federrath, C., Brunt, C., et al. 2011, ApJ, 740, 120

Rosolowsky, E. W., Goodman, A. A., Wilner, D. J., \& Williams, J. P. 1999, ApJ, 524, 887

Rosolowsky, E. W., Pineda, J. E., Kauffmann, J., \& Goodman, A. A. 2008, ApJ, 679, 1338

Sawada, T., Hasegawa, T., Sugimoto, M., Koda, J., \& Handa, T. 2012, ApJ, 752,118

Scalo, J. M. 1984, ApJ, 277, 556

Seabold, S., \& Perktold, J. 2010, in 9th Python in Science Conf., Statsmodels: Econometric and Statistical Modeling with Python, ed. S. van der Walt \& J. Millman (San Fransisco, CA: Scipy), 61

Stanimirović, S., \& Lazarian, A. 2001, ApJL, 551, L53

Stutzki, J., Bensch, F., Heithausen, A., Ossenkopf, V., \& Zielinsky, M. 1998, A\&A, 336, 697

Tessore, N., \& Metcalf, R. B. 2015, A\&A, 580, A79

Tofflemire, B. M., Burkhart, B., \& Lazarian, A. 2011, ApJ, 736, 60

Tsallis, C. 1988, JSP, 52, 479

van der Walt, S., Schönberger, J. L., Nunez-Iglesias, J., et al. 2014, PeerJ, $2, \mathrm{e} 453$

Vazquez-Semadeni, E. 1994, ApJ, 423, 681

Waskom, M., Botvinnik, O., O'Kane, D., et al. 2017, mwaskom/seaborn: v0.8.1 (September 2017), Zenodo, doi:https://doi.org/10.5281/zenodo. 883859

Yeremi, M., Flynn, M., Offner, S., Loeppky, J., \& Rosolowsky, E. 2014, ApJ, 783, 93

Yuen, K. H., \& Lazarian, A. 2017, ApJL, 837, L24

Zielinsky, M., \& Stutzki, J. 1999, A\&A, 347, 630 